\title{
Tissue Microarray
}

National Cancer Institute

\section{Source}

National Cancer Institute. Tissue Microarray. NCI Thesaurus. Code C19922.

A device for use in high-throughput molecular analysis/screening of clinical tissue specimens. Tissue microarrays spatially arrange 500-1000 tumor biopsies or other tissue onto a microarray block, which is then sliced into sections for probing DNA, RNA or protein targets to provide coincident analysis of all specimens in the array. Subsequent sections can be analyzed with additional probes. 